\title{
Comparison of otoacoustic emissions in patients with tinnitus having normal hearing versus mild hearing loss
}

\author{
Fernanda Gentil \\ Susana Meireles ${ }^{2}$ \\ Thuane Roza ${ }^{2}$ \\ Carla Santos ${ }^{2}$ \\ Marco Parente ${ }^{2}$ \\ Eurico Almeida ${ }^{3}$ \\ Renato $\mathrm{Natal}^{2}$
}

\begin{abstract}
Introduction: Tinnitus is an auditory sensation whose source comes from external stimulus to the body. All studies that can help people with this disorder are very imperative. Objective: This study investigates the cochlear function in patients with tinnitus, using Distortion Products Otoacoustic Emissions (DPOAE). Material and Methods: Ears where the subjects referred to feel the tinnitus were considered for the study group while other ears without this sensation of tinnitus acted as a control group. Fifty subjects suffering from unilateral or bilateral tinnitus with normal hearing sensitivity or mild hearing loss were recruited. Results and conclusions: Where comparing the control and study group, the highest percentage of cases of DPOAE detected was in the control group, although these differences were not statistically significant. When the analyzed frequency is the same as the tinnitus frequency, the prevalence of detected DPOAE is in tinnitus ears (50.0\%). In ears where tinnitus was not perceived $(73.5 \%)$ a $p$ value of 0.024 $(<0.05)$ was found, which means that the undetected DPOAE could be influenced by tinnitus. Based on the results, tinnitus might not be caused by changes in the outer hair cells but seems to be affected by that.
\end{abstract}

Keywords: tinnitus, otoacoustic emissions, spontaneous, hearing loss

\footnotetext{
${ }^{1}$ Biomechanics - LAETA, INEGI, Faculdade de Engenharia da Universidade do Porto, Clinica ORL Dr. Eurico Almeida, Widex, Department of Audiology - ESTSP, Porto - AC - Portugal. E-mail: fernanda.fgnanda@gmail.com

2 Biomechanics - LAETA, INEGI, Faculdade de Engenharia da Universidade do Porto - Porto - AC - Portugal. E-mail: susanameiras@gmail.com / thuaneroza@yahoo.com.br / fsantos. carla@gmail.com/mparente@fe.up.pt/rnatal@fe.up.pt

${ }^{3}$ Otorhinolaryngology - Clinica ORL - Dr. Eurico Almeida - Porto - AC - Portugal. E-mail: clinicaorlea@gmail.com

Institution: LAETA, INEGI, Faculdade de Engenharia da Universidade do Porto.

Send correspondence to:

Department of Mechanical Engineering, Faculty of Engineering, University of Porto

Rua Dr. Roberto Frias, s/n, 4200-465 Porto, Portugal. E-mail: fernanda.fgnanda@gmail.com

Paper submitted to the RBCMS-SGP (Publishing Management System) on October 3, 2015;

and accepted on November 11, 2015. cod. 211
} 


\section{INTRODUCTION}

The word tinnitus derives from the Latin tinnire meaning 'to ring'. The current definition of tinnitus is a perception of a sound in the absence of external stimulus. According to Jastreboff, tinnitus is a symptom, not a disease. The tinnitus effects depend on the degree of stimulation of the autonomic and limbic nervous systems and not on the activation level in the auditory pathways ${ }^{1}$.

Against some assumptions that an increase in the firing rate of primary auditory nerve fibers produced by hyperactivity of hair cells inside the cochlea will lead to tinnitus, a decreasehas been noted in the otoacoustic emissions $(\mathrm{OAE})$, rather than the increase expected if the above theory were correct ${ }^{2}$.

Recent developments in the cochlear mechanical process research suggests that one origin of tinnitus could be associated to cochlear mechanical tasks. Damage to the outer hair cells $(\mathrm{OHC})$ of the cochlea is believed to be one of the principal mechanisms of tinnitus $^{1,3}$. There are evidence that non-linear mobile features of the $\mathrm{OHC}$ leads to $\mathrm{OAE}$, through the action of myosin and actin, the elements responsible for contraction in the infrastructure of the $\mathrm{OHC}^{4}$ and the $\mathrm{OHC}$ active mobility seems to be the reason for the frequency selectivity and high hearing sensitivity. The presence of $\mathrm{OAE}$ is a strong evidence of cochlear structural integrity and their lack could indicate a cochlear lesion due the link with $\mathrm{OHC}$ activity ${ }^{5}$.

The assumption that a healthy cochlea may shows low intensity tonal or narrow-band sound when there are not acoustic stimulus was introduced by Gold in $1948^{6}$ who devised a mathematical model of active processes in which the cochlea not only received sounds but also generated acoustic energy. However, it was only in 1977 that David Kemp documented OAE in a normal hearing subject that OAE measurement became possible 7 . Since then, an increased interest in the use of these measures to explore peripheral hearing function in unprecedented depth and detail has been noted. When sound stimulates the cochlea, the $\mathrm{OHC}$ vibrate. The vibration creates an almost inaudible sound that echoes back into the middle ear. The healthy cochlea generates internal vibrations when it processes sound waves. Therefore, OAE is a phenomenon that may appear in normal hearing ears or even in ears with mild hearing loss.

There are different kind of optoacoustic emissions: spontaneous otoacoustic emissions (SOAE), transiently evoked otoacoustic emissions (TEOAE), which is the response to a given auditory stimuli, and distortion product otoacoustic emissions (DPOAE). The essential purpose of an OAE test is to verify cochlear status, mainly $\mathrm{OHC}$ functioning ${ }^{3}$. DPOAE is the unique tool to study cochlear mechanics in humans and animals. The two pure tone stimuli that induce DPOAE have two different frequencies $f_{1}$ and $f_{2}$, identified as primary frequencies. The DPOAE frequencies are algebraic combinations of the frequencies $f_{1}$ and $f_{2}$. Several DPOAE components could be recorded, but the one that has been thoroughly studied in humans is $2 f_{1}-f_{2}$ DPOAE $^{8}$. DPOAE are believed to be the fast, objective, consistent and reproductible measure of the physiological integrity of the $\mathrm{OHC}$ of the cochlea. These phenomena can be recorded in almost all normal ears, and are known to be reduced or absent in ears with hearing loss. DPOAE would be convenient for understanding tinnitus by evaluating the cochlea, especially the $\mathrm{OHC}$, since they allow the analysis of a great spectrum of frequencies, providing a detailed analysis of almost all the cochlea ${ }^{9}$.

There are several risk factors for tinnitus identified in the biomedical literature, such as age, drugs or medication, frequent loud noise exposure, otologic diseases such as otosclerosis, otitis media, presbycusis, sudden deafness, Ménière's disease, and acoustic schwannoma or even associated with hypo or hyperthyroidism, cardiovascular and cerebrovascular disease and lifestyle or behavioral factors such as cigarette smoking and alcohol consumption ${ }^{10}$. The relationship between anxiety and depression and the risk of tinnitus has been reported as well.

The study shows a statistically significant heritability for tinnitus in a sample of 544 monozygotic and samesex dizygotic elderly female twins who contributed to the fourth wave of the Longitudinal Study of Aging Danish Twins ${ }^{11}$. On the other hand, no significant heritability for tinnitus was found in the sample of 412 monozygotic and same-sex dizygotic elderly male twins in this Danish cohort; but the male sample shows that long-term exposure to occupational noise was the main risk factor for tinnitus ${ }^{11}$.

As tinnitus can be associated with hearing loss ${ }^{12}$, the present study was based on the hypothesis that tinnitus without hearing loss or associated with mild hearing loss, can be caused by abnormalities of the central and subcortical level, along with changes in $\mathrm{OHC}$ function.

\section{MATERIAL AND METHODS}

Fifty patients with normal hearing sensitivity or mild hearing loss referring the sensation in the ear of unilateral or bilateral tinnitus were recruited for this study. All patients had "chronic tinnitus" that had lasted for at least 3 months. In patients with unilateral tinnitus, the healthy ear acted as a control ear (normal hearing sensitivity/mild hearing loss without tinnitus) consisting of a total of 34 control ears and 66 tinnitus ears (study group). They were recruited from one Clinic that was approved by the relevant research ethics committee and 
all the individuals who participated in the study signed the Informed Consent Form.

Patients were questioned in order to obtain relevant information about their clinical history. Following this, audiological assessment was performed, including standard pure tone audiometry; discomfort levels (in order to assess the presence of hyperacusis); the research of the pitch and intensity of the tinnitus, and assessment of DPOAE.

Audiological assessment was conducted using the Midimate 622 - Clinical Diagnostic Audiometer (Madsen, Denmark). Based on this test, only patients with normal hearing or with mild hearing loss were selected for this study. The following frequencies were tested: $0.5,1,2$, 3, 4, 6 and $8 \mathrm{kHz}$.

An average tone loss is calculated, taking as a starting point the loss in $\mathrm{dB}$ at following frequencies: 0.5 , 1,2 and $4 \mathrm{kHz}$ (International Organization [ISO]). Normal hearing has an average smaller than 20 decibel hearing level ( $\mathrm{dB} \mathrm{HL})$ and a mild hearing loss corresponds to 21-40 dB HL. In this study, the average \pm standard deviation (SD) of audiograms for normal ears was $17 \pm$ $2.4 \mathrm{~dB} \mathrm{HL}$ and the average $\pm \mathrm{SD}$ for mild hearing loss was $30 \pm 6.6 \mathrm{~dB} \mathrm{HL}$.

DPOAE were measured using the DP-gram Procedure, in response to pure tones of levels ( $L 1=65$ $\mathrm{dB} S P L, L 2=55 \mathrm{~dB} S P L)$ in the frequency range 500$8000 \mathrm{~Hz}$ using a Capella Cochlear Emission Analyzer (Madsen, Denmark). DPOAE were tested for the following frequencies: $0.5,1,2,3,4,6$ and $8 \mathrm{kHz}$.

The statistical analysis was done considering the results in ears, not patients. Average, SD and frequencies were used to describe the results. Normality of distribution was tested with Kolmogorov-Smirnov test. To verify the differences between the tinnitus ears (study group) and ears without the sensation of tinnitus (control group) in regards to DPOAE, the ANOVA test was used. The chi-square test for independence was used to discover if there was a relationship between DPOAE with the two groups. Values with $P \leq 0.05$ were considered statistically significant. All analyses were performed using the statistical software SPSS version 20.0 (SPSS Inc, Chicago, IL, USA).

\section{RESULTS}

\section{Demographic data}

The population consisted of 29 males and 21 females with an age range from 10 to 70 years (average \pm SD of $49.0 \pm 12.9)$. 71 ears had a normal hearing sensitivity and 29 had mild hearing loss. The study group consisted of 66 tinnitus ears and the control group of 34 ears without tinnitus. Tinnitus prevalence was $42.4 \%$ in right ears and $57.6 \%$ in left ears from 66 tinnitus ears. No statistically significant differences were observed between the study group tinnitus ears and the control group normal hearing ears (Table 1 ) in relation to gender, age and auditory acuity, as follows:

Table 1.Tinnitus Patients: Demographic and clinical characteristics.

\begin{tabular}{lccc}
\hline Characteristic & $\begin{array}{c}\text { Control Group } \\
\text { (No tinnitus) }\end{array}$ & $\begin{array}{c}\text { Study Group } \\
\text { (Tinnitus ears) }\end{array}$ & $P$ \\
\hline Ears $(\mathrm{n})$ & 34 & 66 & - \\
Sex ratio (female:male) & $13: 21$ & $29: 37$ & 0.584 \\
Age (mean $\pm \mathrm{SD}$; years) & $48.91 \pm 13.66$ & $48.98 \pm 12.43$ & 0.977 \\
Hearing loss $(\% / \mathrm{n})$ & $17.6(6 / 34)$ & $34.8(23 / 66)$ & 0.073 \\
\hline
\end{tabular}

The proportion of males within the study group $(37 / 66)$ was not significantly different from the proportion of males within the control group (21/34). Since the mean patient age was 48.91 years in the control group and 48.98 in the study group, there was not a statistically significant difference in the mean age for tinnitus and no tinnitus ears. Also, no statistically significant differences were found in the proportion of ears with normal hearing sensitivity between the two groups, although the proportion of them was larger in the control group than in the study group (82.4 vs $65.2 \%$, respectively).

\section{B. Tinnitus frequencies}

Distribution of the perceived tinnitus frequencies in the 66 ears of the study group, obtained from pitchmatching, was also calculated which can be seen in the Figure 1. Observing the histogram of Figure 1, it was possible to identify that the most of the tinnitus was found in the higher frequencies, between 4 and $8 \mathrm{kHz}$.

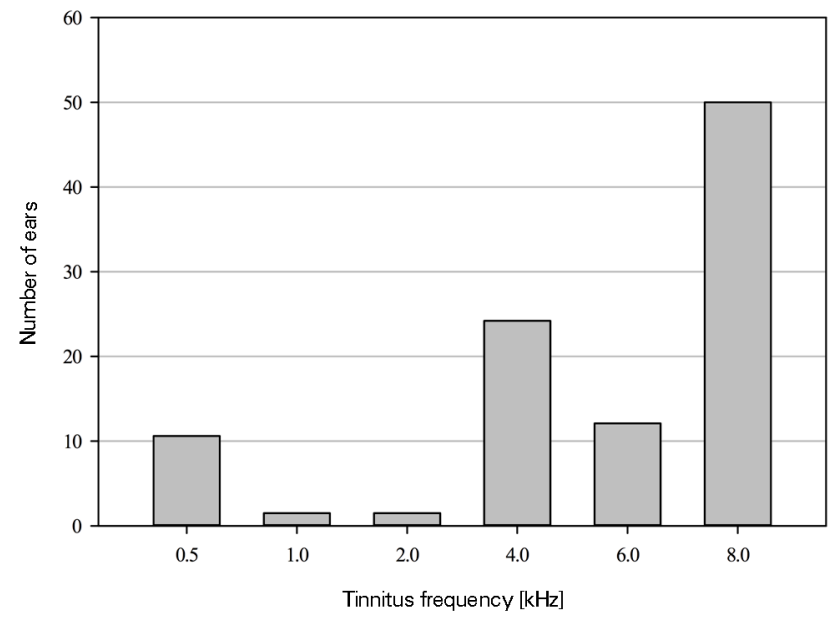

Figure 1. Distribution of tinnitus frequency in the 66 investigated tinnitus ear.

The most common observed frequency of tinnitus was clearly $8 \mathrm{kHz}$, reaching $50 \%$ of all tinnitus ears. The 
middle frequencies were less common with $1.5 \%$ of the sample for 1 and $2 \mathrm{kHz}$ and $10.6 \%$ for $500 \mathrm{~Hz}$. Tinnitus at 4 and $6 \mathrm{kHz}$ of frequency was detected in 24.2 and $12.1 \%$ of the study group, respectively.

\section{Distortion products otoacoustic emissions}

The analysis of DPOAE assessments (Figure 2) showed that it is in the control group (ears without sensation of tinnitus) that the higher percentage of cases where DPOAE were detected for all test frequencies was obtained. The ANOVA test was used for determine differences between the two groups. The Post hoc comparisons using the Tuckey test indicated that the difference was present in the frequency of $3 \mathrm{kHz}$. The results of this test can be observed in Table 2 .

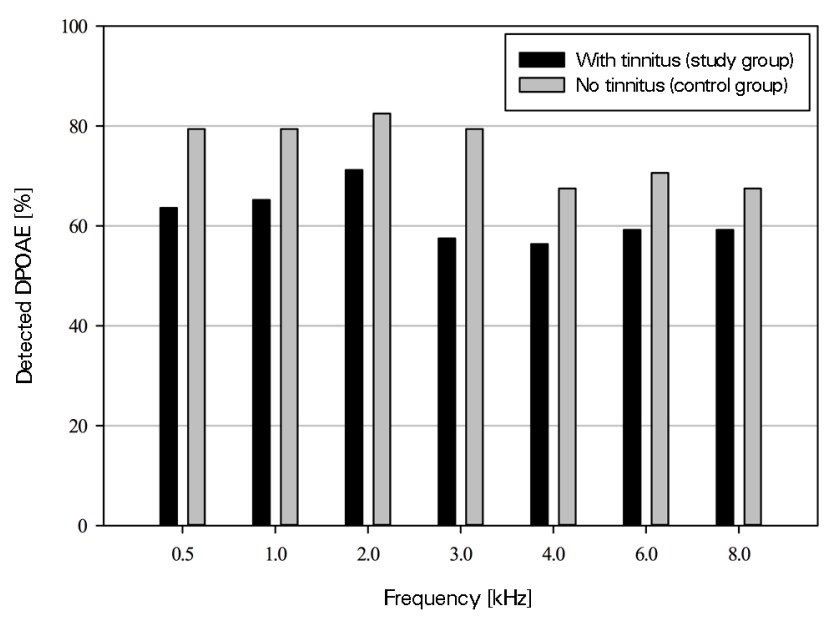

Figure 2. Distribution of detected DPOAE according to the frequencies evaluated in ears.

Table 2. P-values calculated for all test frequencies.

\begin{tabular}{lccccccc}
\hline $\begin{array}{l}\text { DPOAE } \\
\text { detected }(\mathrm{kHz})\end{array}$ & 0.5 & 1 & 2 & 3 & 4 & 6 & 8 \\
\hline$p$ value & 0.108 & 0.143 & 0.227 & 0.030 & 0.267 & 0.264 & 0.409 \\
\hline
\end{tabular}

Based on Table 2, we are able to say that the $p$ value is higher than 0.05 for all frequencies, with exception of $3 \mathrm{kHz}$. So, we accept the null hypothesis $\left(\mathrm{H}_{0}\right)$, which states that the no detected DPOAE is independent of tinnitus. That is, there is insufficient evidence to conclude that the absence or presence of DPOAE influences the occurrence of tinnitus when the analyzed frequency could not be the same as the tinnitus frequency.

The ANOVA test was also used to verify if there are differences with the results of the audiograms, and the values obtained where $P=0.074$ and $F=3.266$. Then, we can conclude that there are no significant differences between subjects with normal hearing and subjects with mild hearing loss, when the two groups are compared (with and without sensation of tinnitus).

Therefore, it is important to check what happens when the analyzed frequency is the same as the tinnitus frequency.

The chi-square for independence test to test $\mathrm{H}_{0}$, which states that the undetected DPOAE is independent of tinnitus, was used. In this statistical test only the results for each tinnitus frequency were used. Statistically significant differences were found in the proportion of ears with tinnitus that did not show DPOAE in relation to those without tinnitus that did not show DPOAE, as we can see in the Table 3.

Table 3. Percentage of no detected and detected DPOAE* according to each group by using chi-square for independence test.

\begin{tabular}{lccc}
\hline Group & $\begin{array}{c}\text { No detected } \\
\text { DPOAE }\end{array}$ & $\begin{array}{c}\text { Detected } \\
\text { DPOAE }\end{array}$ & P-value \\
\hline Control Ears $(\mathrm{N}=34)$ & $26.5 \%$ & $73.5 \%$ & 0.024 \\
Tinnitus Ears $(\mathrm{N}=66)$ & $50.0 \%$ & $50.0 \%$ & \\
\hline
\end{tabular}

* Distortion Products Otoacoustic Emissions

The $P$ value is lower than a, so we reject $\mathrm{H}_{0}$. That is, rejecting the null hypothesis suggests that the alternative hypothesis may be true, which means that the undetected DPOAE could be dependent of tinnitus.

\section{DISCUSSION}

There are different hypotheses regarding mechanisms of tinnitus generation. Most of the authors consider the origin of tinnitus as cochlear, like lesions of the hair cells after acoustic trauma, presbycusis, sudden hearing loss, etc. Tinnitus was perceived as a sound, and the receptor organ of sound was the ear, so this leads that a significant proportion of tinnitus cases are also related with the onset of an auditory pathology, such as otitis media, acoustic trauma, sudden deafness, etc. ${ }^{13}$. The current theory on tinnitus generation is based upon Jastreboff's "neurophysiological model"1,14 and postulates that tinnitus is a consequence of a modified neural activity in the central auditory system consequential to a peripheral damage in the auditory structures.

The theory of tinnitus centred on neurophysiology propose that all auditory systems, and some no auditory systems particularly the limbic system (involved in emotion), and the autonomic nervous system, which controls all body functions, are an essential part of the most cases of tinnitus. According to this model the annoyance of tinnitus is determined exclusively by the limbic and autonomic nervous systems. A weak imbalance of neuronal activity within the auditory 
system, is noticed at low levels in the auditory system, and being a new signal it is enhanced by subcortical centres, transferred to the auditory cortex and perceived as a sound - tinnitus, and evaluated. In the majority of the cases, the continued presence of tinnitus combined without any situation associated results in habituation of the reaction to the tinnitus signal ${ }^{1}$.

Jastreboff ${ }^{1}$ considered that tinnitus frequently starts in the cochlea and then the abnormal activity is generated in the central pathways that prolong the symptoms.

The present study intends to test if the cochlear dysfunction has been implicated in tinnitus generation. The OAE are always associated with the behavior of $\mathrm{OHC}$, their presence is an indicator of cochlear structural integrity and on the other hand their lack could indicate a cochlear lesion ${ }^{5}$. DPOAE are reduced or absent when the mobility of the OHC, which is the source of OAE generation, is impaired by damage to those cells ${ }^{15}$. Some previous studies demonstrated that DPOAE incidence greatly differed depending on age ${ }^{16}$ gender and hearing level.

Relating the tinnitus prevalence with age and using 1990 Hearing Supplement data, Ries ${ }^{17}$ reported that from the 15 million adults with bothersome tinnitus, about 27 $\%$ have more than 65 years old. Though, more than 41 $\%$ were under 45 years. There are more young adults affected by tinnitus than seniors in spite of the double increase of prevalence for seniors. Tinnitus prevalence levels between 65 and 74 years and then declines progressively. It is well known that hearing thresholds are minor in adult men than in women and that thresholds get worse with age in both genders.

In the same way, DPOAE amplitudes have been found to be larger in younger subjects and in women than in men ${ }^{18}$. Reduction in OAE is known to follow decreased task of the stria vascularis and to follow hair cell loss ${ }^{16}$. Consequently, as audiometric thresholds worsen, the amplitude of the DPOAE response decreases and finally disappears ${ }^{19}$. Cilento et al. ${ }^{16}$ shown that women lose DPOAE amplitude from age and hearing losses, with men loosing more amplitude than women. Cilento et al. also concluded that the loss in amplitude is related to the degree of hearing loss.

Several studies have attempted to study the aging effect on the DPOAE amplitude ${ }^{20,21}$. However, these results may have been influenced by the effect of hearing loss since it is challenging to isolate the aging consequence from that. For these reasons, the significance of the difference between the two groups considered in the present study, in relation to female-male ratio, average age and normal hearing/mild hearing loss ratio, were obtained in order to confirm that both groups did not have significant differences in relation to those aspects. As the results show, no significant differences were found between the two groups in respect of gender, age or auditory acuity (Table 1).

Since recent reports suggest that one source of tinnitus may be related to cochlear mechanical activity, the present work intends to study the presence/absence of DPOAE in tinnitus ears with normal hearing sensitivity or mild hearing loss. In this study, $73.5 \%$ of DPOAE presence was found among ears with normal hearing sensitivity or mild hearing loss, without tinnitus (control group). Ears with normal hearing or mild hearing loss in combination with tinnitus (study group) showed a lower incidence than the control group, reaching only $50 \%$ of total ears, when ears were compared at coincident frequencies between DPOAE and tinnitus (Table 3).

In the present study the differences observed between the prevalence of detected DPOAE in tinnitus ears and that in ears without tinnitus were considered statistically significant, which means that the significance was lower than or equal to 0.05 (Chi-square for independence test, $P=0.024)$. So the absence of DPOAE could not be determined independently of tinnitus. Based on these results, tinnitus could have not been caused by changes in $\mathrm{OHC}$ but seems be affected by that.

Based on previous reports and in accordance with the results found in the present study, we can say that whatever the etiology, at the same levels of hearing thresholds, DPOAE seems to be lower when chronic tinnitus is present ${ }^{22-26}$. Liu et al..$^{24}$ analyzed the association between OAE and tinnitus in 306 ears with tinnitus (with or without hearing loss) and observed that in $94.8 \%$ of those with hearing loss and tinnitus the DPOAE-gram shows lower amplitude or was absent within the frequency range of elevated pure-tone-threshold. In $59 \%$ of cases with normal hearing and tinnitus, the amplitude of DPOAE at almost all frequencies of tinnitus was decreased. At other frequencies than that of tinnitus, the amplitude of DPOAE was normal.

Shiomi et al. ${ }^{25}$ studied cochlear activity in 9 tinnitus patients who had normal hearing and 55 who had also hearing impairment, and compared both groups to a control one with 26 normal-hearing individuals without any symptoms of tinnitus. Substantial decreases in DPOAE amplitude over a frequency range between $4-7 \mathrm{kHz}$ were obtained in $93.3 \%$ of the tinnitus ears in the normalhearing group and in $96 \%$ of the tinnitus ears in the other group, while just $15.4 \%$ in the control group. In that study, although hearing thresholds did not significantly differ between tinnitus and nontinnitus normal-hearing subjects, DPOAE amplitudes of tinnitus patients were significantly lower than individuals without tinnitus over $4-7 \mathrm{kHz}$ range, which suggests that the decrease of DPOAE amplitudes over a limited frequency range may be associated with the occurrence of tinnitus. 
Dall'Igna et al. ${ }^{27}$ investigated about the OAE in patients with symptoms of tinnitus and normal hearing. The results showed that $60 \%$ had OAE absent or lowered in one or more frequencies. Others studies also shown that $O A E$ were worse in ears of tinnitus individuals with normal hearing ${ }^{3,22,28,29}$ suggesting abnormalities of cochlear micromechanics in these ears, even without hearing loss. Qasem et al. ${ }^{3}$ tried to compare cochlear $\mathrm{OHC}$ function between 48 normal hearing patients, half with tinnitus and the other half without tinnitus. They found significant decreases in the DPOAE amplitudes over the following frequencies ranges $750-6000 \mathrm{~Hz}$ in all tinnitus patients compared to nontinnitus, although hearing threshold levels did not shown significant differences differ between both groups. This implies that the decrease of DPOAE amplitudes may be related to the occurrence of tinnitus.

Granjeiro et al. ${ }^{30}$ tested the hypothesis that tinnitus begins with OHC dysfunction by recording DPOAE in 32 tinnitus patients with normal hearing and 37 patients without tinnitus but similar thresholds. In this paper DPOAE were anomalous in $68.4 \%$ of the tinnitus patients and in $50 \%$ of the healthy ones, showing that the study group had significantly higher prevalence of abnormal DPOAE than the control one.

Figure 1 of the present study shows that a higher incidence of tinnitus was found for a frequency range between 4 and $8 \mathrm{kHz}$, reaching an incidence of $83 \%$ of all ears analyzed. It was in this frequency range that the most reduced incidence of DPOAE detected was found for both tinnitus and nontinnitus ears (Figure 2). Together with this information, we have noticed, based on the same figure that no significant differences were found between tinnitus ears and nontinnitus ears at each test frequency, including the lower frequencies and even at higher frequencies. It can be concluded that the reduced number of ears with detected DPOAE at high frequencies was probably related to other reason than tinnitus.

The presence of tinnitus, with or even without hearing loss, has also been shown to alter DPOAE ${ }^{9}$. Some studies reported that a tinnitus patient seems to have lower DPOAE than nontinnitus patients ${ }^{9,22,23,25,29,31,32}$. However, many of these studies included patients with poor hearing thresholds and with tinnitus, and this could possibly influence the results. Hearing loss is usually associated with reduced DPOAE amplitude, making difficult to conclude whether the perceived reduction in DPOAE amplitude was associated to tinnitus alone or instead was just due to the hearing loss. Other studies reported increased DPOAE amplitude ${ }^{33,34}$ but again, the involved subjects in these studies presented differences in the hearing thresholds, what does not clarify if the irregularities in DPOAE are due to the expansion of tinnitus or due to the hearing losses ${ }^{32}$.
Until now, a few studies have investigated DPOAE by including tinnitus patients with normal audiometric thresholds $9,22,29,30-32$.

Quite different results were found by Gouveris et al. ${ }^{33}$ who compared the cochlear $\mathrm{OHC}$ function by DPOAE-grams of 32 ears with acute tonal tinnitus and normal hearing or minimal hearing loss with 17 healthy nontinnitus ears. They show that tinnitus ears presented relatively increased amplitudes of DPOAE at high frequencies which range between $4-6.3 \mathrm{kHz}$ when compared with the group of healthy ears and relatively decreased DPOAE amplitudes at middle frequencies from 1650 to $2400 \mathrm{~Hz}$, while the one that present statistically significant increased average values of DPOAE amplitudes have been detected at $4.9 \mathrm{kHz}$.

Based on that, they suggested that a cochlear lesion results in a decreased activity of the $\mathrm{OHC}$.

Sztuka et al. ${ }^{34}$ measured the features of DPOAE in a 44 tinnitus patients group without hearing loss and compared to 33 individuals without tinnitus and normal hearing sensibility (control group). In their study, the average DPOAE amplitudes for frequencies of 3003 , 4004, and $5005 \mathrm{~Hz}$ in patients with tinnitus and proper hearing were higher than in the control group. These results were consistent with Gouviers et al. ${ }^{33}$ but not in accordance with the present study and studies referred above $3,9,23-25,27,29,31$ which reported lower DPOAE amplitudes in cases with tinnitus than in cases without tinnitus. Sztuka et al. ${ }^{34}$ similarly to Gouveris et al. ${ }^{33}$ recognized the increase in amplitude of the DPOAE to an increased movement of the OHCs induced by reduced neural activity in the auditory nerve.

Differently from what happened in all the surveys listed above, Singh et al. ${ }^{35}$ who studied 25 normal-hearing patients with tinnitus and 20 patients with normal hearing and no tinnitus. They did not discover any statistical differences between the DPOAE amplitude of the ears with tinnitus versus the control ears, although slightly increased DPOAE amplitudes were detected for tinnitus ears. They also compared the DPOAE signal-to-noise ratio between the two groups and found that individuals with normal hearing and with tinnitus had significantly more abnormal DPOAE results than individuals with normal hearing but without tinnitus. Their clarification would be the different levels of higher auditory pathways to the dysfunction of the $\mathrm{OHC}$. We would agree with Jastreboff, who indicated that the levels of the auditory pathway may be involved in the creation of tinnitus, but, in the majority of cases, the cause is probably at the OHC level'.

Paglialonga et al. ${ }^{32}$ studied the probable role played by both $\mathrm{OHC}$ and cochlear efferent system functionality in tinnitus ears with normal hearing. The work of the team shown the occurrence of abnormal OAEs, 
mainly at higher frequencies, in patients with tinnitus and with normal hearing, but no correlation was obtained concerning the range of frequencies with abnormal DPOAE level and the bandwidth of tinnitus (tonal, narrow-band, or broadband) or, in patients with tonal tinnitus, with the pitch. Furthermore significantly lower DPOAE, mainly at high test frequencies were measured in subjects with tinnitus compared to nontinnitus controls, reflecting $\mathrm{OHC}$ dysfunction generally in high-frequency cochlear regions.

Although others reason than tinnitus could be possibly involved in the reduced DPOAE, different results seems to be found in the present study, since no significant differences were found between tinnitus ears and nontinnitus ears at all test frequencies when DPOAEs were compared between the two groups at each frequency independently of the tinnitus frequency (Table 2). The significance level increased to a significant value when the results were compared at coincident frequencies between DPOAEs and tinnitus as referred above (Table 3 ), suggesting that there was some correlation between the frequencies range with no detected DPOAE. It is noted that the audiometer and the Capella Cochlear Emission Analyzer, Madsen used only measure frequencies below $8 \mathrm{kHz}$. Probably we should evaluate frequencies above $8 \mathrm{kHz}$. This can be considered one limitation of this study.

This study has shown that DPOAE has a potential value in the assessment of tinnitus, providing information on the structural integrity of the cochlea, especially $\mathrm{OHC}$ functioning. The results of the present study suggested that it is still premature to conclude that DPOAE provided an objective evidence of tinnitus, considering the number of variables at play. However, it was clear that at least tinnitus seems be affected by the $\mathrm{OHC}$ functioning. So that, more detailed analyses is required to clarify these arguments.

\section{REFERENCES}

1. Jastreboff PJ. Phantom auditory perception (tinnitus): mechanisms of generation and perception. Neurosci Res. 1990;8(4):221-54. DOI: http://dx.doi.org/10.1016/0168-0102(90)90031-9

2. Kiang NY, Liberman MC, Levine RA. Auditory-nerve activity in cats exposed to ototoxic drugs and high-intensity sounds. Ann Otol Rhinol Laryngol. 1976;85(6 PT. 1):752-68. DOI: http://dx.doi. org/10.1177/000348947608500605

3. Qasem H, Assaf $S$, Nawaf AB, Hroot A, Tubishi K, Husban A, et al. Otoacoustic emissions and tinnitus in normal hearing. J R Med Serv. 2010;17(2):27-31.

4. Zenner HP. Motile responses in outer hair cells. Hear Res. 1986;22:83-90. PMID: 3733547 DOI: http://dx.doi.org/10.1016/03785955(86)90082-1

5. Ceranic BJ, Prasher DK, Raglan E, Luxon LM. Tinnitus after head injury: evidence from otoacoustic emissions. J Neurol Neurosurg Psychiatry. 1998;65(4):523-9. PMID: 9771778 DOI: http://dx.doi. org/10.1136/jnnp.65.4.523
6. Gold T. Hearing. II. The physical basis of the action of the cochlea. Proc R Soc Edinb. 1948;135:492-8. DOI: http://dx.doi.org/10.1098/ rspb.1948.0025

7. Zhang T, Penner MJ. A new method for the automated detection of spontaneous otoacoustic emissions embedded in noisy data. Hear Res. 1998;117(1-2):107-13. PMID: 9557981 DOI: http://dx.doi. org/10.1016/S0378-5955(97)00212-8

8. Moulin A, Kemp DT. Multicomponent acoustic distortion product otoacoustic emission phase in humans. II. Implications for distortion product otoacoustic emissions generation. J Acoust Soc Am. 1996;100(3):1640-62. PMID: 8817892 DOI: http://dx.doi. org/10.1121/1.416064

9. Onishi ET, Fukuda Y, Suzuki FA. Distortion product otoacoustic emissions in tinnitus patients. Int Tinnitus J. 2004;10(1):13-6.

10. Hoffman HJ, Reed GW. Epidemiology of tinnitus. In: Snow JB, ed. Tinnitus Theory and Management. London: Decker; 2004. p.16-41.

11. Petersen HC, Andersen T, Frederiksen H, Hoffman HJ, Christensen K, editors. The heritability of tinnitus: a twin study; Poster presented at: Nordic Epidemiology Congress; June 9-12, 2002; Aarhus, Denmark. In: Snow JB. Tinnitus: Theory and Management, Hamilton, London: BC Deckerlnc; 2004.

12. Lockwood AH, Salvi RJ, Burkard RF. Tinnitus. N Engl J Med 2002;347(12):904-10. PMID: 12239260 DOI: http://dx.doi. org/10.1056/NEJMra013395

13. Herraiz C. Physiopathological mechanisms in tinnitus generation and persistence. Acta Otorrinolaringol Esp. 2005;56(8):33542. PMID: 16285431 DOI: http://dx.doi.org/10.1016/S0001. 6519(05)78626-X

14. Jastreboff PJ, Hazell JW. Tinnitus retraining therapy: implementing the neurophysiological model. New York: Cambridge University Press; 2007. p.41-52.

15. Whitehead ML, Lonsbury-Martin BL, Martin GK. Evidence for two discrete sources of 2f1-f2 distortion-product otoacoustic emission in rabbit. II: Differential physiological vulnerability. J Acoust Soc Am. 1992;92(5):2662-82. DOI: http://dx.doi.org/10.1121/1.404382

16. Cilento BW, Norton SJ, Gates GA. The effects of aging and hearing loss on distortion product otoacoustic emissions. Otolaryngol Head Neck Surg. 2003;129(4):382-9. PMID: 14574293 DOI: http://dx.doi. org/10.1016/S0194-5998(03)00637-5

17. Ries PW. Prevalence and characteristics of persons with hearing trouble: United States, 1990-1991. Vital and health statistics, 10 (188). Hyattsville: National Center for Health Statistics; 1994.

18. McFadden D. A speculation about the parallel ear asymmetries and sex differences in hearing sensitivity and otoacoustic emissions. Hear Res. 1993;68(2):143-51. PMID: 8407600 DOI: http://dx.doi. org/10.1016/0378-5955(93)90118-K

19. Martin GK, Ohlms LA, Franklin DJ, Harris FP, Lonsbury-Martin BL. Distortion product emissions in humans. III. Influence of sensorineural hearing loss. Ann Otol Rhinol Laryngol Suppl. 1990;147:30-42.

20. Dorn PA, Piskorski P, Keefe DH, Neely ST, Gorga MP. On the existence of an age/threshold/frequency interaction in distortion product otoacoustic emissions. J Acoust Soc Am. 1998;104(2 Pt 1):964-71. DOI: http://dx.doi.org/10.1121/1.423339

21. Strouse AL, Ochs MT, Hall JW 3rd. Evidence against the influence of aging on distortion-product otoacoustic emissions. J Am Acad Audiol. 1996;7(5):339-45.

22. Castello E. Distortion products in normal hearing patients with tinnitus. Boll Soc Ital Biol Sper. 1997;73(5-6):93-100. PMID: 9796127

23. Kowalska S, Sułkowski W. Tinnitus in noise-induced hearing impairment. Med Pr. 2001;52(5):305-13. PMID: 11828843

24. Liu B, Liu C, Song B. Otoacoustic emissions and tinnitus. Zhonghua Er Bi Yan Hou Ke Za Zhi. 1996;31(4):231-3.

25. Shiomi Y, Tsuji J, Naito Y, Fujiki N, Yamamoto N. Characteristics of DPOAE audiogram in tinnitus patients. Hear Res. 1997;108(12):83-8. PMID: 9213125 DOI: http://dx.doi.org/10.1016/S03785955(97)00043-9 
26. Job A, Cian C, Esquivié D, Leifflen D, Trousselard M, Charles C, et al. Moderate variations of $\mathrm{mood} / \mathrm{emotional}$ states related to alterations in cochlear otoacoustic emissions and tinnitus onset in young normal hearing subjects exposed to gun impulse noise. Hear Res. 2004;193(1-2):31-8. PMID: 15219318 DOI: http://dx.doi. org/10.1016/j.heares.2004.02.010

27. Dall Igna C, Schmidt LP, Smith M, Facchini L, Kang S. Otoacoustic emissions in patients with tinnitus and normal hearing. Otolaryngol Head Neck Surg. 2004;131(2):279. DOI: http://dx.doi.org/10.1016/j. otohns.2004.06.593

28. McKee GJ, Stephens SD. An investigation of normally hearing subjects with tinnitus. Audiology. 1992;31(6):313-7. DOI: http:// dx.doi.org/10.3109/00206099209072919

29. Ozimek E, Wicher A, Szyfter W, Szymiec E. Distortion product otoacoustic emission (DPOAE) in tinnitus patients. J Acoust Soc Am. 2006;119(1):527-38. PMID: 16454306 DOI: http://dx.doi. org/10.1121/1.2141297

30. Granjeiro RC, Kehrle HM, Bezerra RL, Almeida VF, Sampaio AL, Oliveira CA. Transient and distortion product evoked oto-acoustic emissions in normal hearing patients with and without tinnitus. Otolaryngol Head Neck Surg. 2008;138(4):502-6. PMID: 18359362 DOI: http://dx.doi.org/10.1016/j.otohns.2007.11.012
31. Ami M, Abdullah A, Awang MA, Liyab B, Saim L. Relation of distortion product otoacoustic emission with tinnitus. Laryngoscope. 2008;118(4):712-7. PMID: 18176342 DOI: http://dx.doi.org/10.1097/ MLG.0b013e318161e521

32. Paglialonga A, Del Bo L, Ravazzani P, Tognola G. Quantitative analysis of cochlear active mechanisms in tinnitus subjects with normal hearing sensitivity: multiparametric recording of evoked otoacoustic emissions and contralateral suppression. Auris Nasus Larynx. 2010;37(3):291-8. DOI: http://dx.doi.org/10.1016/j. anl.2009.09.009

33. Gouveris $\mathrm{H}$, Maurer J, Mann W. DPOAE-grams in patients with acute tonal tinnitus. Otolaryngol Head Neck Surg. 2005;132(4):550-3. PMID: 15806043 DOI: http://dx.doi.org/10.1016/j.otohns.2004.09.031

34. Sztuka A, Pospiech L, Gawron W, Dudek K. DPOAE in estimation of the function of the cochlea in tinnitus patients with normal hearing. Auris Nasus Larynx. 2010;37(1):55-60. DOI: http://dx.doi. org/10.1016/j.anl.2009.05.001

35. Singh S, Munjal SK, Panda NK. Comparison of auditory electrophysiological responses in normal-hearing patients with and without tinnitus. J Laryngol Otol. 2011;125(7):668-72. DOI: http:// dx.doi.org/10.1017/S0022215111000569 